Supplement of Hydrol. Earth Syst. Sci., 24, 3835-3850, 2020

https://doi.org/10.5194/hess-24-3835-2020-supplement

(c) Author(s) 2020. This work is distributed under

the Creative Commons Attribution 4.0 License.

(c) (1)

Hydrology and
Earth System
Sciences

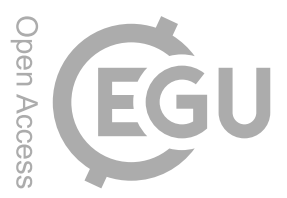

Supplement of

\title{
Survival of the Qaidam mega-lake system under mid-Pliocene climates and its restoration under future climates
}

\author{
Dieter Scherer \\ Correspondence to: Dieter Scherer (dieter.scherer@tu-berlin.de)
}

The copyright of individual parts of the supplement might differ from the CC BY 4.0 License. 


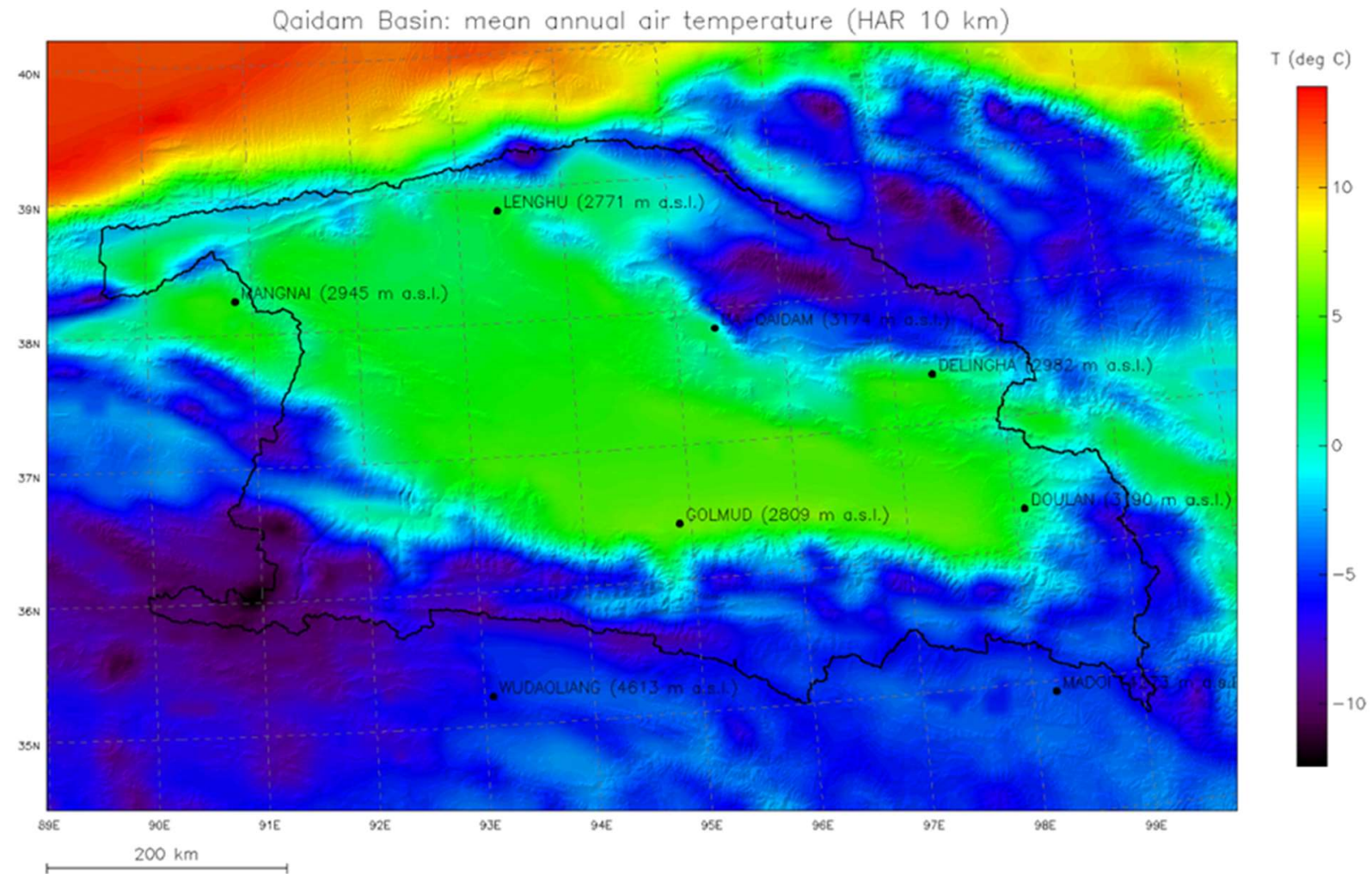

Figure S1: Mean annual air temperature ( $2 \mathrm{~m}$ above ground) during the study period of 14 hydrological years (2001-2014) for the Qaidam Basin (QB) and its surrounding regions derived from the HAR $10 \mathrm{~km}$ data set. Black line: boundary of the QB (Lehner and 5 Grill, 2013). Topographic shading is based on DEM data from the SRTM. Black dots indicate the locations of the eight GSOD stations within or nearby the QB. 


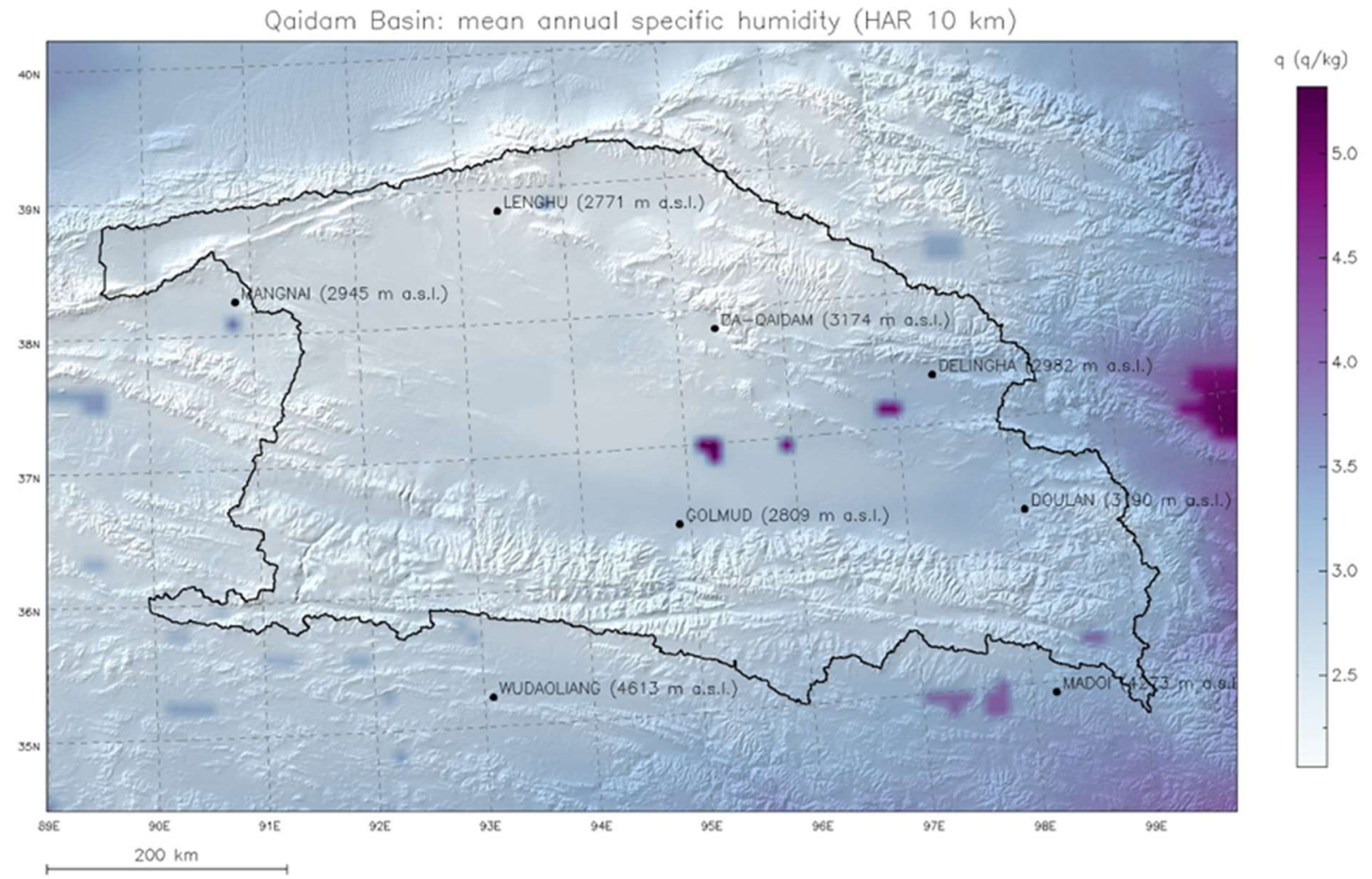

Figure S2: Mean annual specific humidity ( $2 \mathrm{~m}$ above ground) during the study period of 14 hydrological years (2001-2014) for the Qaidam Basin (QB) and its surrounding regions derived from the HAR $10 \mathrm{~km}$ data set. Black line: boundary of the QB (Lehner and 10 Grill, 2013). Topographic shading is based on DEM data from the SRTM. Black dots indicate the locations of the eight GSOD stations within or nearby the $\mathrm{QB}$. 


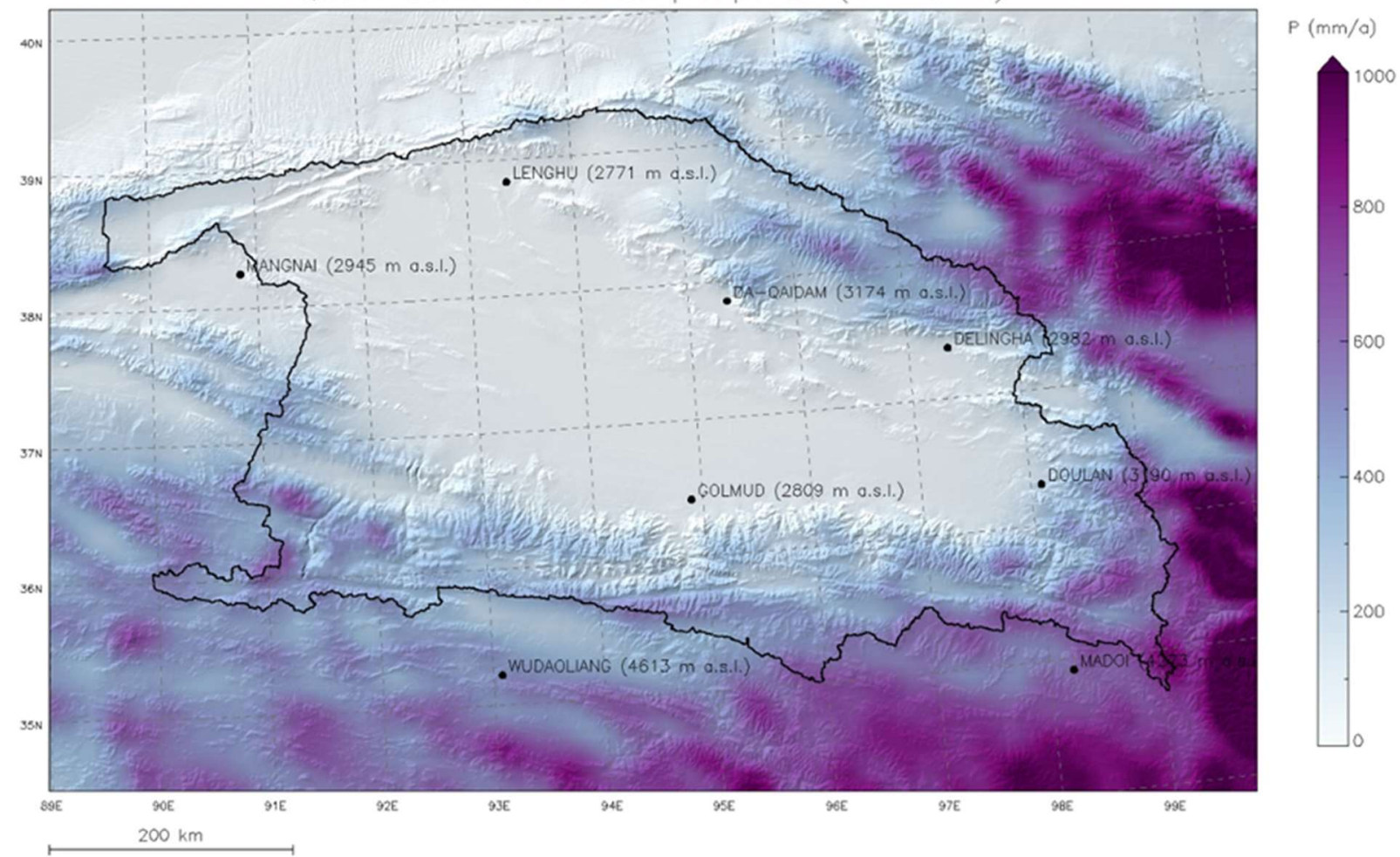

Figure S3: Mean annual precipitation during the study period of 14 hydrological years (2001-2014) for the Qaidam Basin (QB) and its surrounding regions derived from the HAR $10 \mathrm{~km}$ data set. Black line: boundary of the $Q B$ (Lehner and Grill, 2013). Topographic 15 shading is based on DEM data from the SRTM. Black dots indicate the locations of the eight GSOD stations within or nearby the QB. 


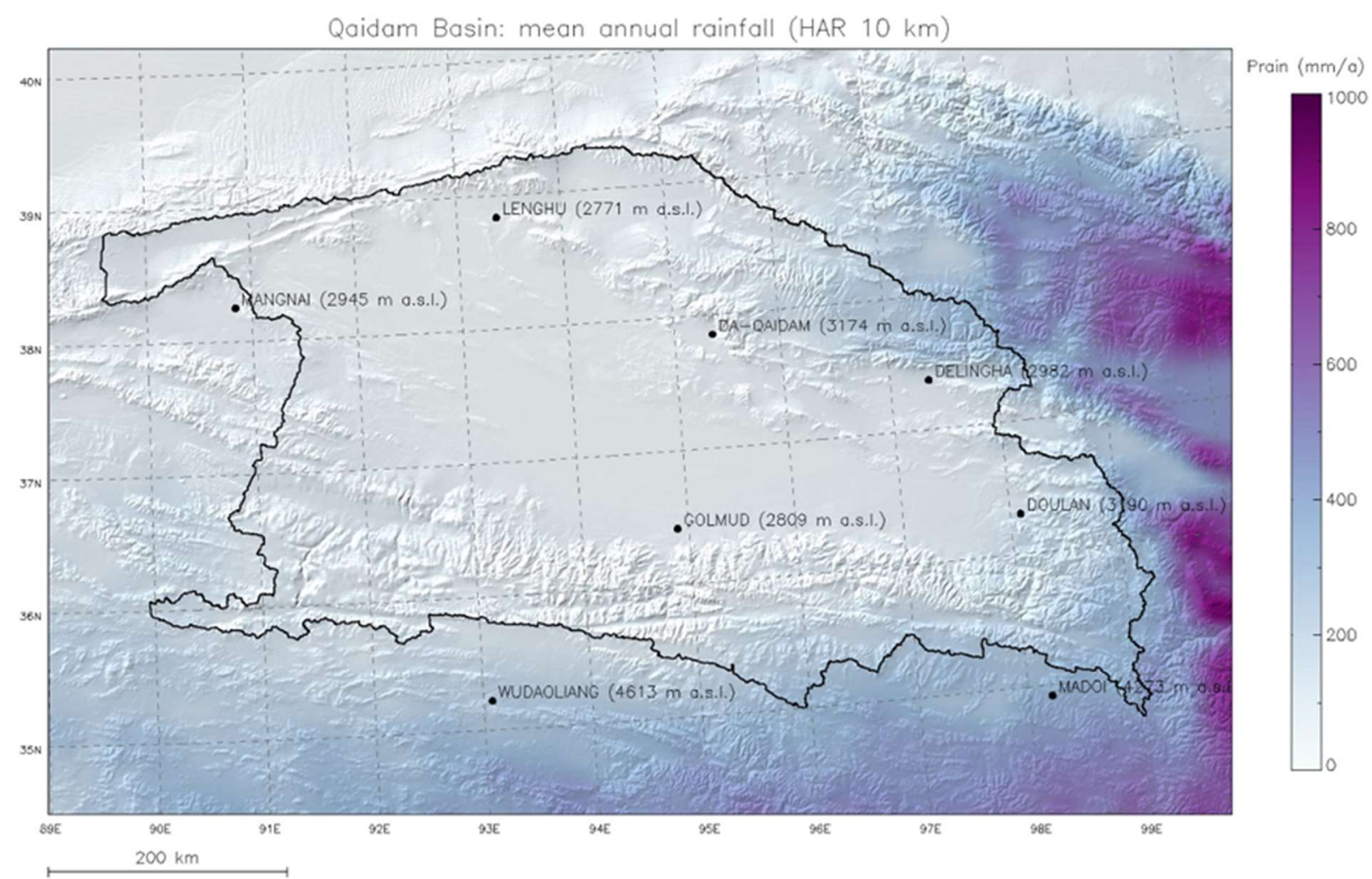

Figure S4: Mean annual rainfall during the study period of 14 hydrological years (2001-2014) for the Qaidam Basin (QB) and its surrounding regions derived from the HAR $10 \mathrm{~km}$ data set. Black line: boundary of the QB (Lehner and Grill, 2013). Topographic shading is based on DEM data from the SRTM. Black dots indicate the locations of the eight GSOD stations within or nearby the QB. 


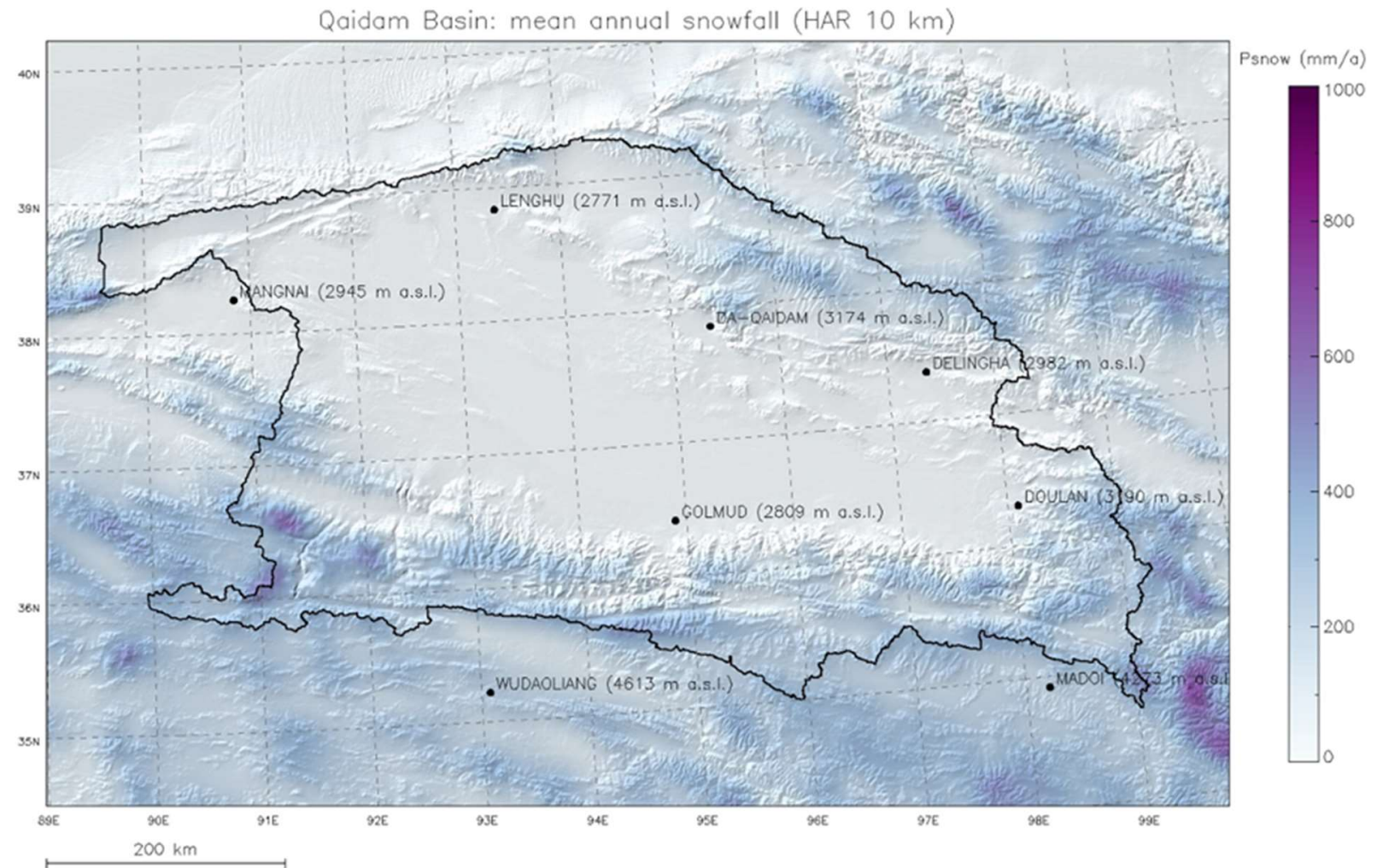

Figure S5: Mean annual snowfall during the study period of 14 hydrological years (2001-2014) for the Qaidam Basin (QB) and its surrounding regions derived from the HAR $10 \mathrm{~km}$ data set. Black line: boundary of the QB (Lehner and Grill, 2013). Topographic shading is based on DEM data from the SRTM. Black dots indicate the locations of the eight GSOD stations within or nearby the QB. 


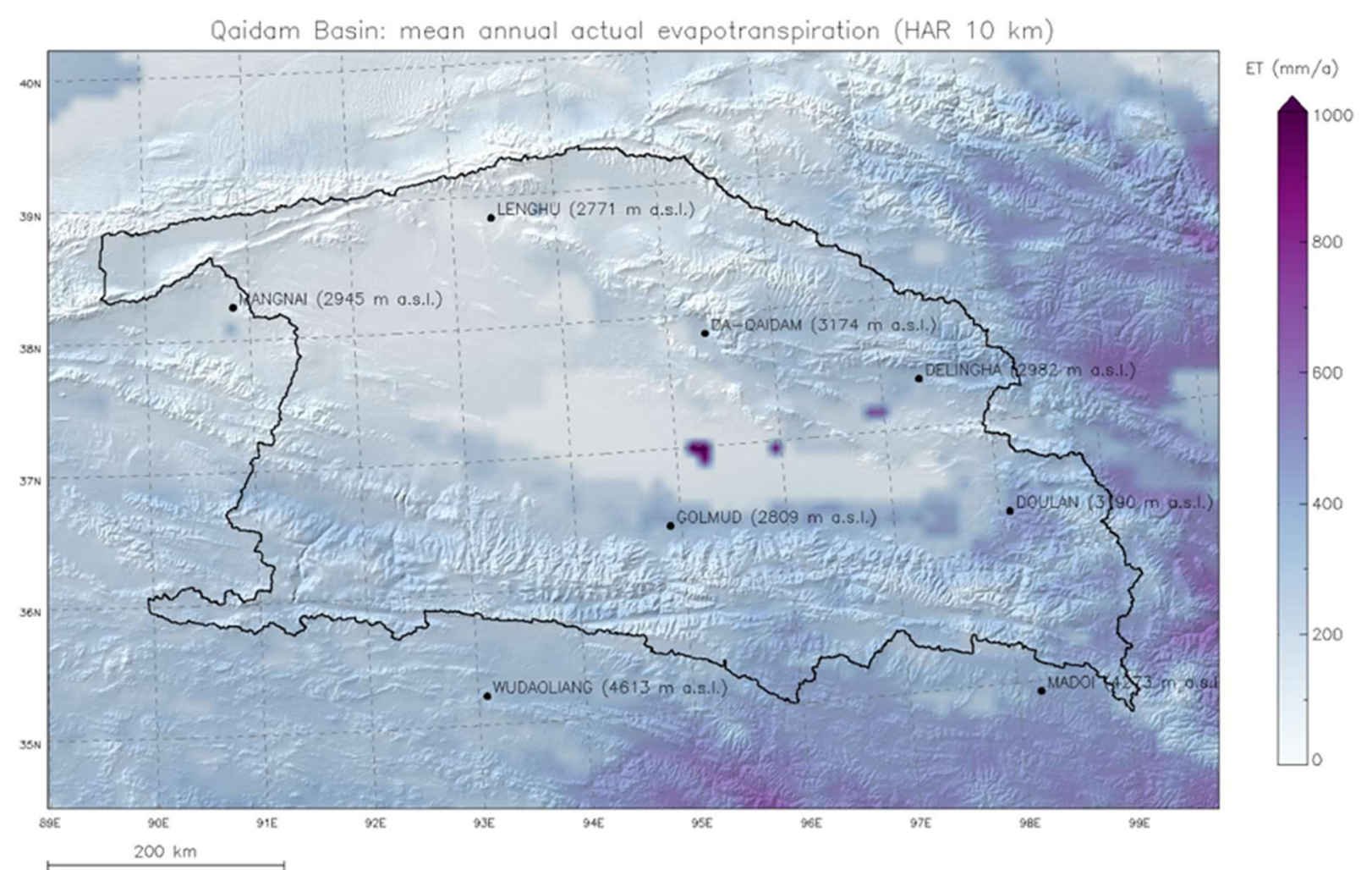

Figure S6: Mean annual actual evapotranspiration during the study period of 14 hydrological years (2001-2014) for the Qaidam Basin (QB) and its surrounding regions derived from the HAR $10 \mathrm{~km}$ data set. Black line: boundary of the QB (Lehner and Grill,

30 2013). Topographic shading is based on DEM data from the SRTM. Black dots indicate the locations of the eight GSOD stations within or nearby the $Q B$. 


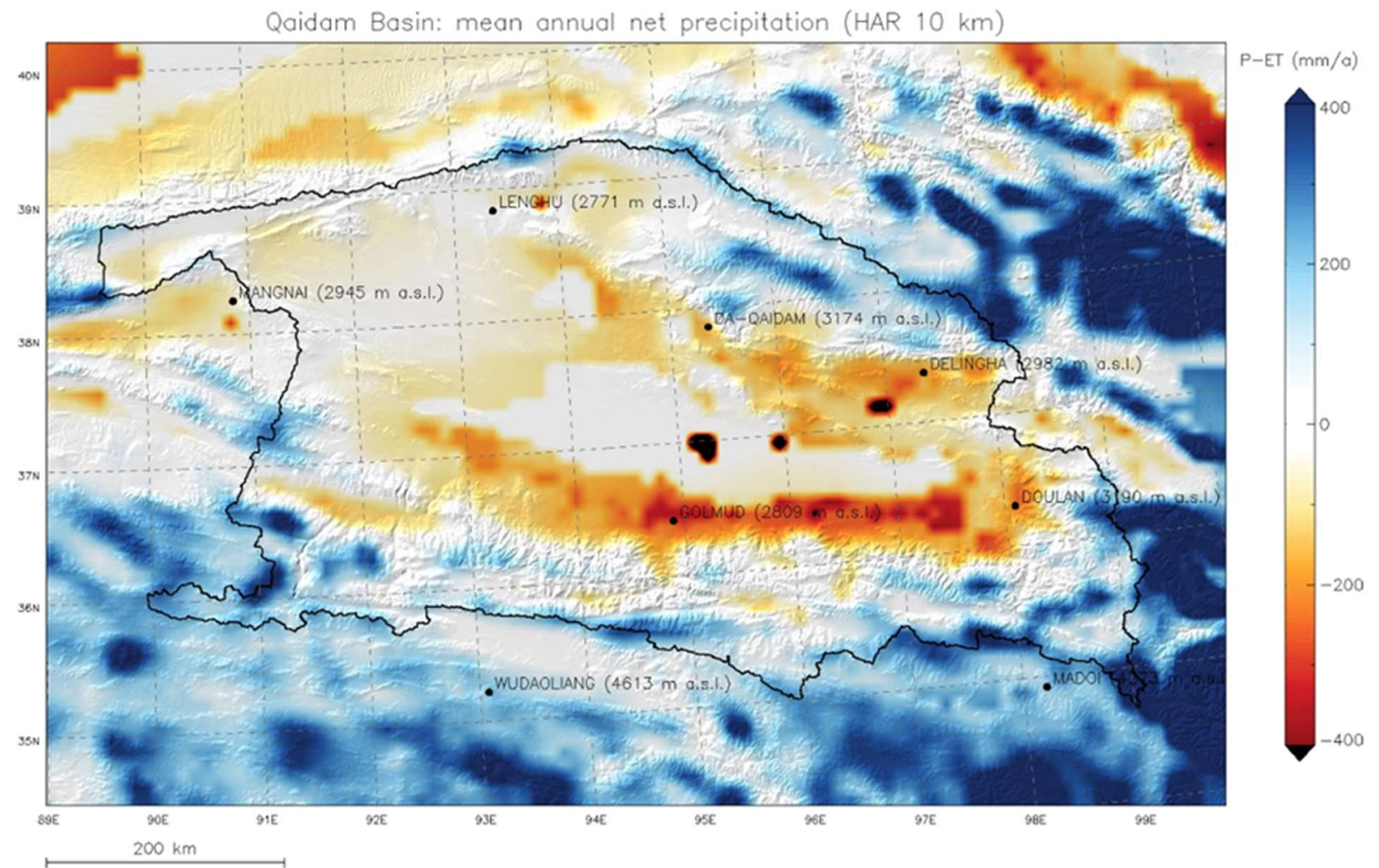

Figure S7: Mean annual net precipitation, i.e., precipitation $P$ minus actual evapotranspiration $E T$, during the study period of 14 hydrological years (2001-2014) for the Qaidam Basin $(Q B)$ and its surrounding regions derived from the HAR $10 \mathrm{~km}$ data set. Black 35 line: boundary of the QB (Lehner and Grill, 2013). Topographic shading is based on DEM data from the SRTM. Black dots indicate the locations of the eight GSOD stations within or nearby the QB. 


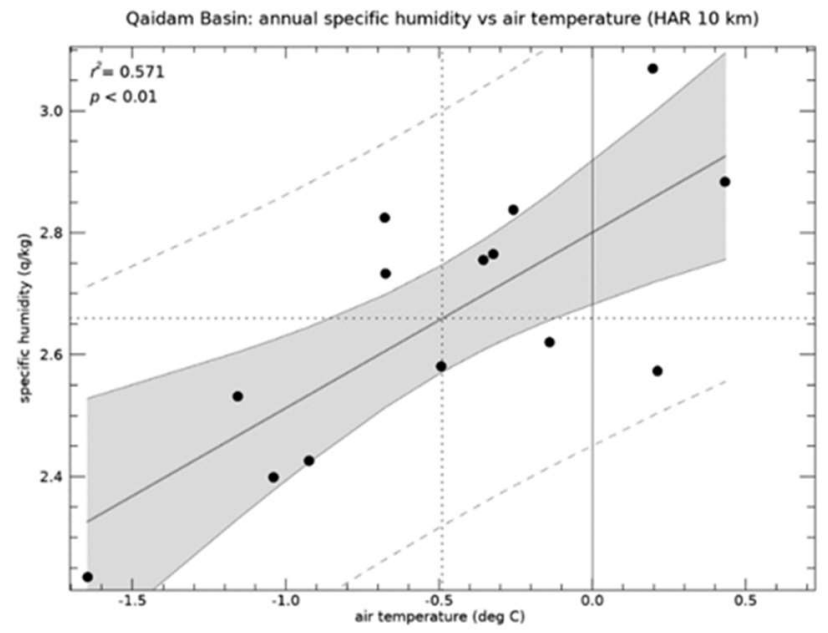

Figure S8: Specific humidity versus air temperature in the Qaidam Basin (QB) during the hydrological years 2001 to 2014 derived from the HAR $10 \mathrm{~km}$ data set. Dotted lines: mean annual values; solid lines: regression lines; light grey shades: confidence interval; dashed lines: prediction interval.
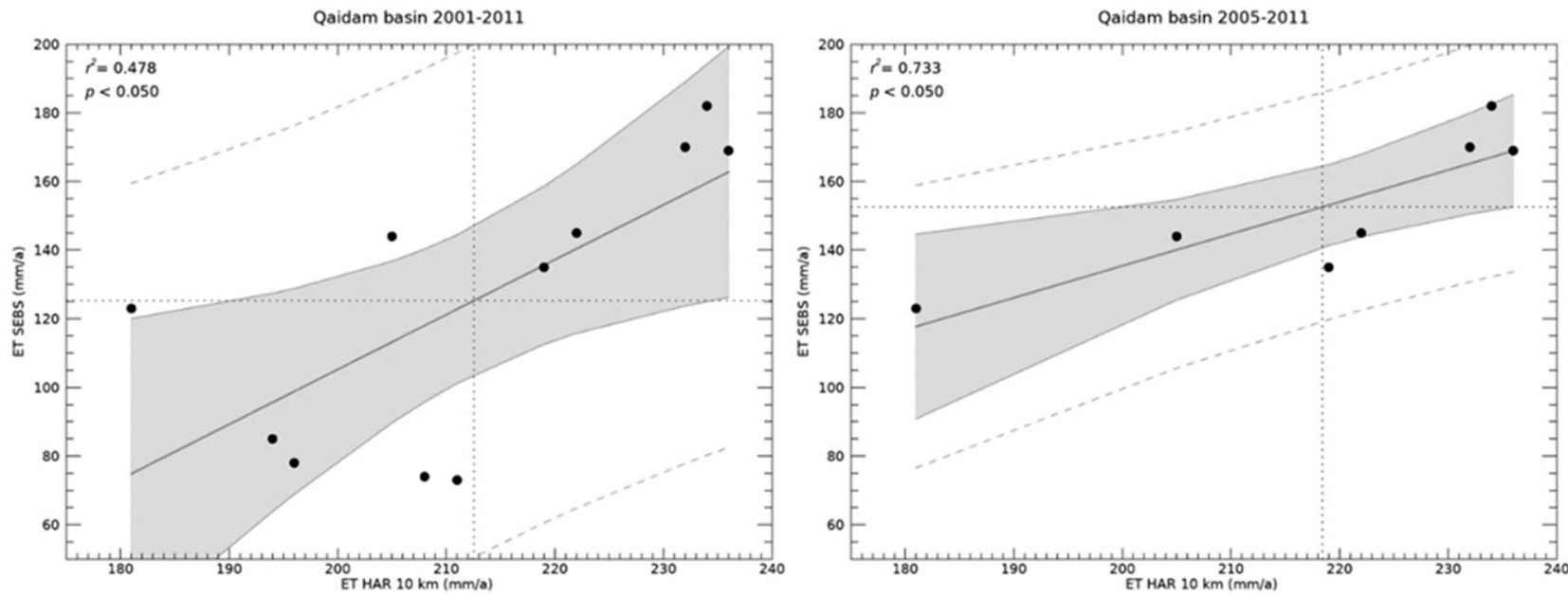

Figure S9: Annual actual evapotranspiration ET determined by the SEBS-based study of Jin et al. (2013) versus ET of the HAR $10 \mathrm{~km}$ data set in the Qaidam Basin (QB) for the calendar years 2001-2011 (left panel) and 2005-2011 (right panel). Dotted lines: mean annual values; solid lines: regression lines; light grey shades: confidence intervals; dashed lines: prediction intervals. 

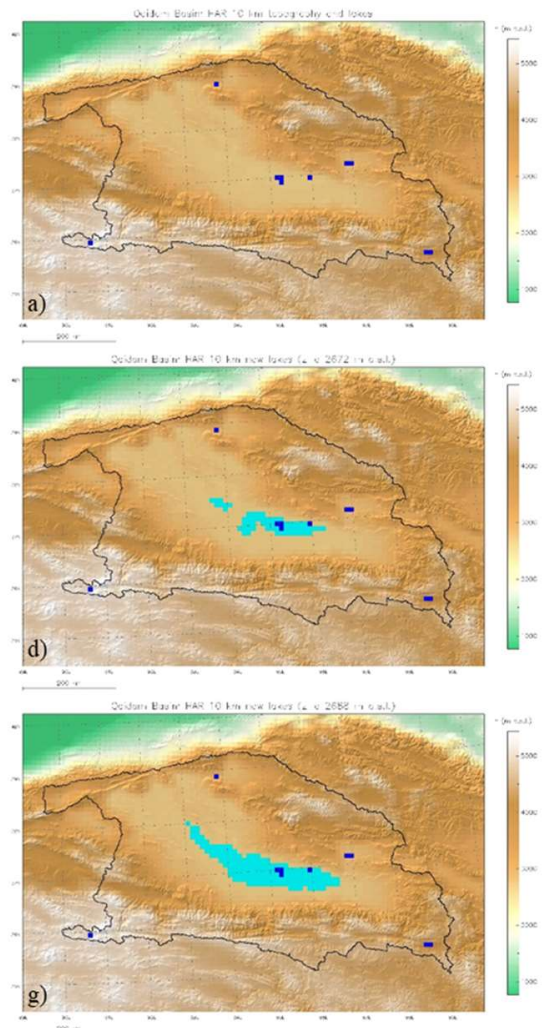
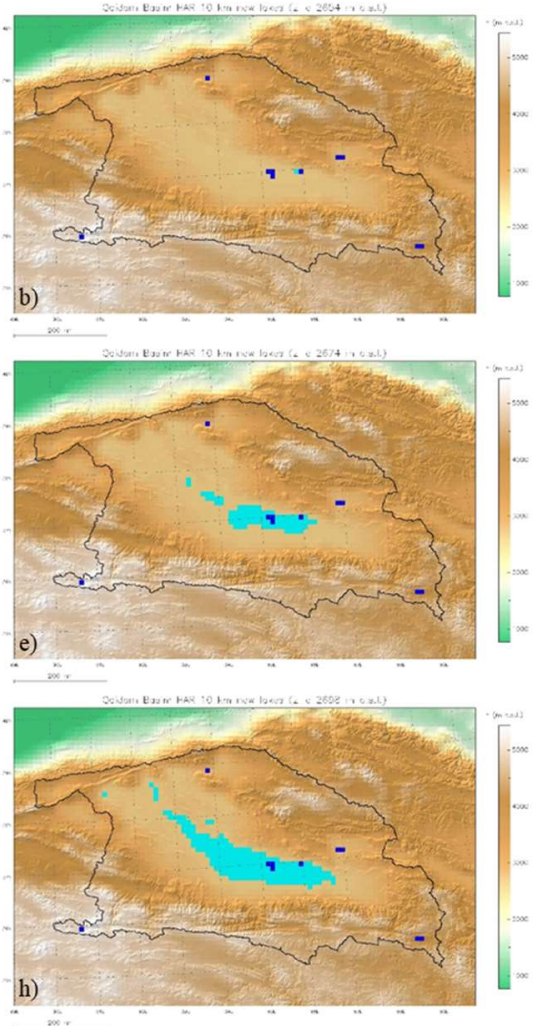
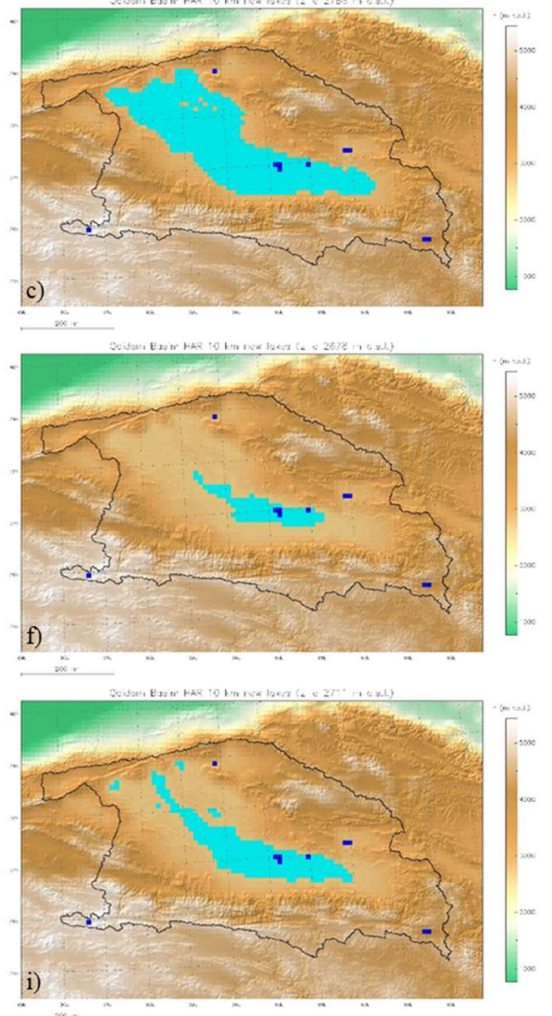

Figure S10: Panel a): present-day lake extent in the Qaidam Basin (QB) as represented in the HAR $10 \mathrm{~km}$ data set. Panels b) to i): illustrations of lake extents for different projections of equilibrium lake states using the present-day model topography of the

50 HAR $10 \mathrm{~km}$ data set for accumulation of net precipitation in the $Q B$ and subsequent runoff originating from land in areas below equilibrium lake levels (marked in cyan). Equilibrium lake levels (cf. Table 3): panel b) $2654 \mathrm{~m}$ a.s.l. (one metre above lowest presentday level); panel c) maximum extent of the mega-lake system of approx. $59000 \mathrm{~km}^{2}$ as reported by Chen and Bowler (1986); 2786 m a.s.l.; panel d) $2672 \mathrm{~m}$ a.sl. $\left(d P_{Q B}=50 \mathrm{~mm} / \mathrm{a} ; E T_{\text {lake }}=1000 \mathrm{~mm} / \mathrm{a}\right)$; panel e) $2674 \mathrm{~m}$ a.sl. $\left(d P_{Q B}=50 \mathrm{~mm} / \mathrm{a} ; E T_{\text {lake }}=800 \mathrm{~mm} / \mathrm{a}\right)$; panel f) $2678 \mathrm{~m}$ a.sl. $\left(d P_{Q B}=50 \mathrm{~mm} / \mathrm{a} ; E T_{\text {lake }}=600 \mathrm{~mm} / \mathrm{a}\right)$; panel g) $2688 \mathrm{~m}$ a.sl. $\left.\left(d P_{Q B}=100 \mathrm{~mm} / \mathrm{a} ; E T_{\text {lake }}=1000 \mathrm{~mm} / \mathrm{a}\right) ; \mathrm{panel} \mathrm{h}\right)$

$552698 \mathrm{~m}$ a.sl. $\left(d P_{Q B}=100 \mathrm{~mm} / \mathrm{a} ; E T_{\text {lake }}=800 \mathrm{~mm} / \mathrm{a}\right)$; panel i) $2711 \mathrm{~m}$ a.sl. $\left(d P_{Q B}=100 \mathrm{~mm} / \mathrm{a} ; E_{\text {lake }}=600 \mathrm{~mm} / \mathrm{a}\right)$. Black line: boundary of the QB (Lehner and Grill, 2013). Blue: present-day lake extent $\left(1000 \mathrm{~km}^{2}\right)$ as represented in the HAR $10 \mathrm{~km}$ data set. Topographic shading is based on DEM data from the SRTM. 

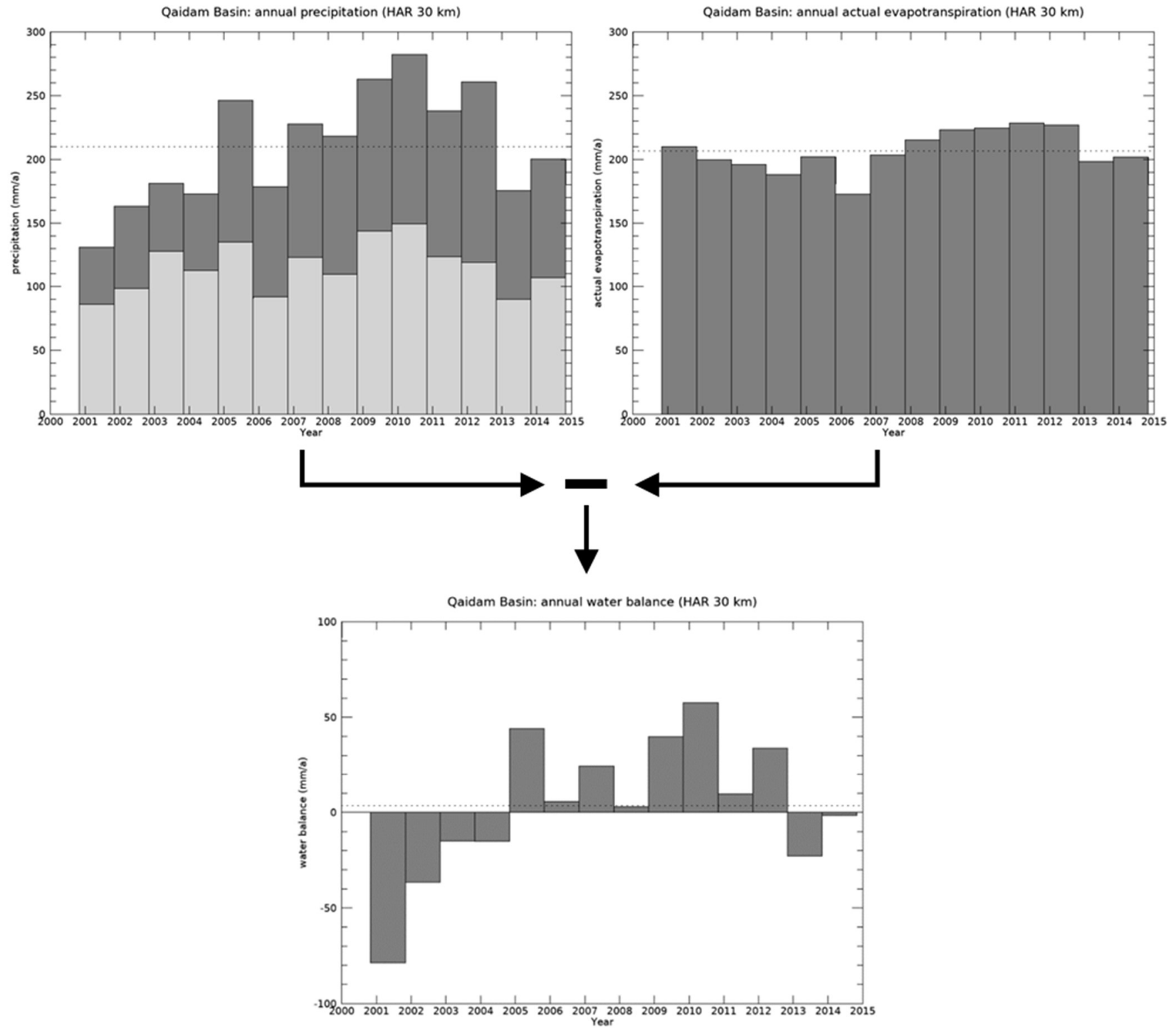

Figure S11: Annual precipitation (upper left panel), actual evapotranspiration (upper right panel), and water balance (lower panel) in the Qaidam Basin (QB) during the hydrological years 2001 to 2014 as in Fig. 2 but derived from the HAR $30 \mathrm{~km}$ data set. Upper left panel: light grey bars: annual snowfall; dark grey bars: annual rainfall. Dotted lines: mean annual values. 

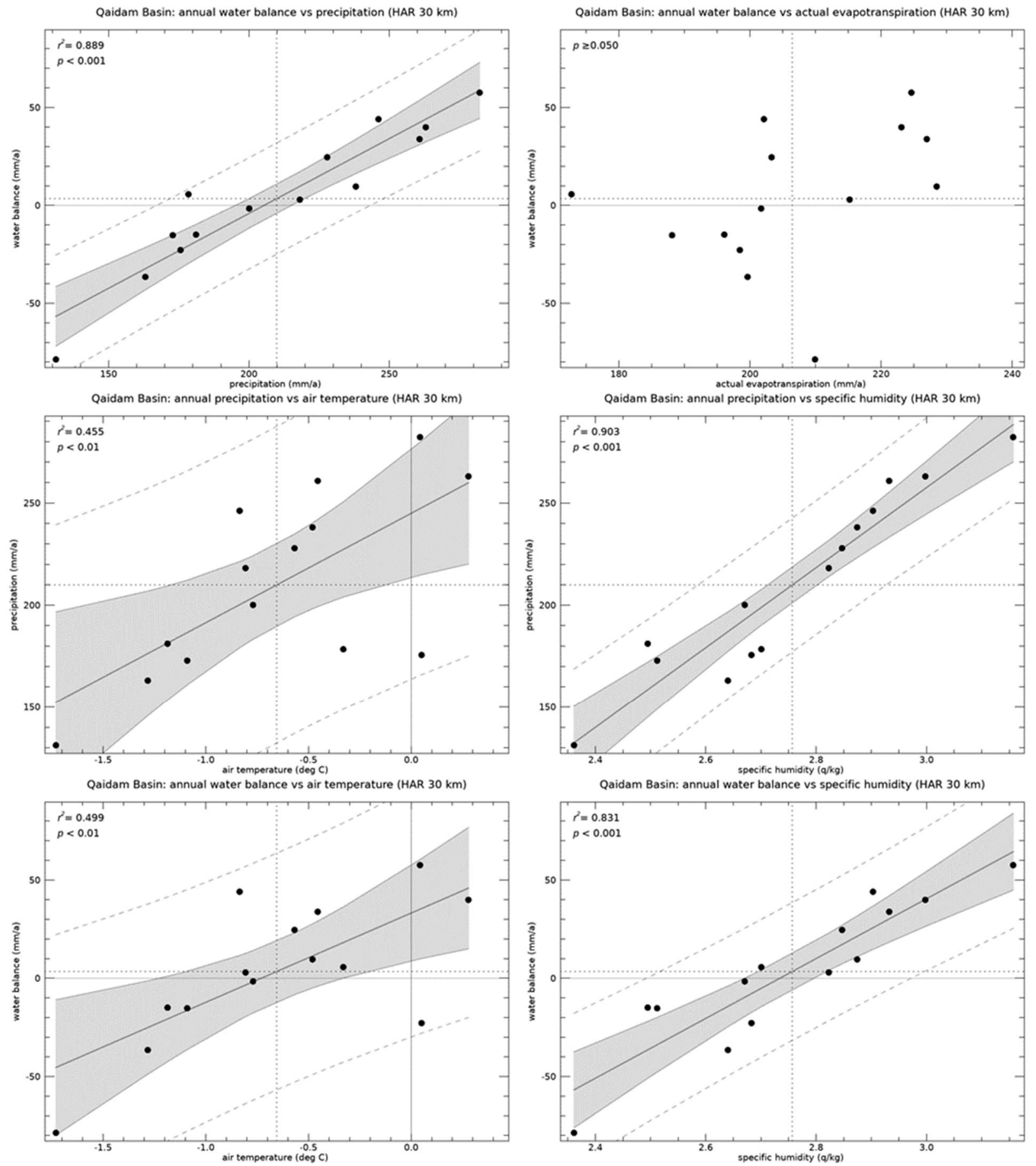

Figure S12: Water balance versus precipitation (upper left panel) and actual evapotranspiration (upper right panel); precipitation versus air temperature (middle left panel) and specific humidity (middle right panel); water balance versus air temperature (lower left panel) and specific humidity (lower right panel) in the Qaidam Basin (QB) during the hydrological years 2001 to 2014 as in Fig. 4 but derived from the HAR $30 \mathrm{~km}$ data set. Dotted lines: mean annual values; solid lines: regression lines; light grey shades: confidence intervals; dashed lines: prediction intervals. 


\section{Supplementary Tables}

Table S2: Monthly and annual air temperature $T$ (in $\operatorname{deg} C$ ) in the Qaidam Basin (QB) during the 14 hydrological years (2001-2014) covered by the HAR $10 \mathrm{~km}$ data set.

\begin{tabular}{|c|c|c|c|c|c|c|c|c|c|c|c|c|c|}
\hline$T$ & 10 & 11 & 12 & 1 & 2 & 3 & 4 & 5 & 6 & 7 & 8 & 9 & year \\
\hline 2001 & -2.0 & -10.3 & -14.2 & -16.1 & -11.5 & -7.7 & -1.8 & 3.5 & 9.4 & 13.3 & 10.6 & 6.5 & -1.6 \\
\hline 2002 & -1.5 & -8.3 & -14.5 & -16.7 & -10.8 & -6.6 & 1.0 & 4.1 & 9.9 & 12.4 & 11.6 & 5.1 & -1.2 \\
\hline 2003 & -3.1 & -9.5 & -12.3 & -13.2 & -10.2 & -5.9 & 0.2 & 3.9 & 9.2 & 11.2 & 10.6 & 6.1 & -1.0 \\
\hline 2004 & -1.2 & -7.4 & -12.7 & -14.9 & -11.6 & -4.3 & 1.4 & 4.1 & 8.4 & 11.2 & 10.3 & 5.3 & -0.9 \\
\hline 2005 & -2.0 & -10.6 & -11.8 & -13.1 & -10.4 & -4.8 & -0.5 & 5.1 & 9.6 & 11.6 & 10.9 & 7.3 & -0.7 \\
\hline 2006 & -0.9 & -8.7 & -13.4 & -11.5 & -8.5 & -6.6 & -0.7 & 5.1 & 9.7 & 13.0 & 12.9 & 7.7 & -0.1 \\
\hline 2007 & 0.8 & -7.3 & -13.7 & -16.2 & -10.0 & -5.8 & 0.9 & 7.0 & 9.4 & 11.5 & 12.0 & 6.6 & -0.4 \\
\hline 2008 & -0.6 & -7.2 & -11.7 & -14.8 & -14.9 & -5.4 & 0.1 & 7.1 & 10.0 & 11.7 & 9.8 & 7.2 & -0.7 \\
\hline 2009 & 0.3 & -7.3 & -11.0 & -12.8 & -8.4 & -5.0 & 2.6 & 5.3 & 10.2 & 12.1 & 10.7 & 8.0 & 0.4 \\
\hline 2010 & -0.6 & -8.2 & -12.6 & -11.5 & -8.9 & -5.3 & -0.8 & 5.4 & 9.8 & 13.9 & 12.8 & 7.7 & 0.2 \\
\hline 2011 & -0.2 & -8.1 & -14.3 & -16.5 & -8.8 & -7.7 & 0.8 & 6.4 & 10.7 & 12.9 & 12.8 & 7.7 & -0.3 \\
\hline 2012 & 0.9 & -5.6 & -12.3 & -17.9 & -11.9 & -6.8 & 0.1 & 6.4 & 10.7 & 12.7 & 12.6 & 7.6 & -0.3 \\
\hline 2013 & -1.0 & -9.1 & -13.7 & -15.2 & -9.6 & -2.5 & 1.3 & 6.3 & 11.5 & 12.9 & 13.8 & 7.4 & 0.2 \\
\hline 2014 & 0.8 & -10.3 & -15.2 & -14.1 & -11.3 & -4.4 & -0.1 & 5.0 & 10.2 & 13.4 & 11.6 & 7.9 & -0.5 \\
\hline mean & -0.7 & -8.4 & -13.1 & -14.6 & -10.5 & -5.6 & 0.3 & 5.3 & 9.9 & 12.4 & 11.6 & 7.0 & -0.5 \\
\hline
\end{tabular}

Table S2: Monthly and annual specific humidity $q$ (in $\mathrm{g} / \mathrm{kg}$ ) in the Qaidam Basin (QB) during the 14 hydrological years (2001-2014) covered by the HAR $10 \mathrm{~km}$ data set.

\begin{tabular}{crrrrrrrrrrrrr}
\hline $\boldsymbol{q}$ & 10 & 11 & 12 & 1 & 2 & 3 & 4 & 5 & 6 & 7 & 8 & 9 & year \\
\hline 2001 & 1.9 & 1.2 & 1.0 & 0.8 & 0.9 & 1.0 & 1.8 & 2.3 & 3.1 & 4.2 & 4.7 & 3.8 & $\mathbf{2 . 2}$ \\
2002 & 1.7 & 1.3 & 1.0 & 0.9 & 1.1 & 1.4 & 2.2 & 2.7 & 4.0 & 5.5 & 4.4 & 4.0 & $\mathbf{2 . 5}$ \\
2003 & 2.0 & 1.4 & 1.3 & 1.1 & 1.4 & 1.7 & 2.4 & 2.5 & 3.5 & 4.0 & 4.3 & 3.1 & $\mathbf{2 . 4}$ \\
2004 & 1.8 & 1.6 & 1.1 & 1.1 & 1.3 & 1.7 & 1.9 & 2.7 & 3.3 & 4.5 & 5.0 & 2.9 & $\mathbf{2 . 4}$ \\
2005 & 2.0 & 1.4 & 1.3 & 1.1 & 1.2 & 1.9 & 1.9 & 2.8 & 4.2 & 6.2 & 5.7 & 4.0 & $\mathbf{2 . 8}$ \\
2006 & 2.0 & 1.2 & 0.8 & 1.1 & 1.5 & 1.1 & 1.7 & 2.4 & 4.3 & 6.4 & 5.1 & 3.5 & $\mathbf{2 . 6}$ \\
2007 & 2.2 & 1.5 & 1.0 & 0.7 & 1.2 & 1.7 & 2.0 & 2.5 & 4.4 & 5.5 & 5.9 & 4.3 & $\mathbf{2 . 8}$ \\
2008 & 2.7 & 1.3 & 1.0 & 1.0 & 1.1 & 1.5 & 1.8 & 3.0 & 4.2 & 6.1 & 4.6 & 4.5 & $\mathbf{2 . 7}$ \\
2009 & 2.6 & 1.6 & 1.2 & 1.1 & 1.3 & 1.5 & 2.0 & 2.9 & 4.1 & 6.1 & 4.8 & 5.3 & $\mathbf{2 . 9}$ \\
2010 & 2.4 & 1.4 & 1.1 & 1.1 & 1.4 & 1.7 & 2.0 & 3.1 & 5.6 & 7.1 & 5.1 & 4.7 & $\mathbf{3 . 1}$ \\
2011 & 2.6 & 1.3 & 0.9 & 0.7 & 1.3 & 1.3 & 2.0 & 3.2 & 4.8 & 5.4 & 5.6 & 4.0 & $\mathbf{2 . 8}$ \\
2012 & 2.3 & 1.7 & 0.9 & 0.8 & 1.2 & 1.5 & 1.7 & 3.4 & 5.0 & 6.5 & 6.0 & 3.1 & $\mathbf{2 . 8}$ \\
2013 & 1.9 & 1.1 & 0.9 & 0.9 & 1.2 & 1.2 & 1.6 & 3.2 & 4.5 & 5.9 & 5.2 & 3.3 & $\mathbf{2 . 6}$ \\
2014 & 2.0 & 1.2 & 0.9 & 0.8 & 1.1 & 1.5 & 2.0 & 2.1 & 4.6 & 5.4 & 5.0 & 4.2 & $\mathbf{2 . 6}$ \\
\hline mean & $\mathbf{2 . 2}$ & $\mathbf{1 . 4}$ & $\mathbf{1 . 0}$ & $\mathbf{0 . 9}$ & $\mathbf{1 . 2}$ & $\mathbf{1 . 5}$ & $\mathbf{1 . 9}$ & $\mathbf{2 . 8}$ & $\mathbf{4 . 3}$ & $\mathbf{5 . 6}$ & $\mathbf{5 . 1}$ & $\mathbf{3 . 9}$ & $\mathbf{2 . 7}$ \\
\hline
\end{tabular}


Table S3: Monthly and annual precipitation $P$ (in millimetres per month or millimetres per year) in the Qaidam Basin (QB) during the 14 hydrological years (2001-2014) covered by the HAR $10 \mathrm{~km}$ data set.

\begin{tabular}{crrrrrrrrrrrrr}
\hline $\boldsymbol{P}$ & 10 & 11 & 12 & 1 & 2 & 3 & 4 & 5 & 6 & 7 & 8 & 9 & year \\
\hline 2001 & 5 & 4 & 6 & 3 & 3 & 3 & 19 & 16 & 17 & 11 & 20 & 15 & $\mathbf{1 2 2}$ \\
2002 & 2 & 1 & 6 & 3 & 3 & 10 & 16 & 25 & 34 & 25 & 12 & 22 & $\mathbf{1 6 0}$ \\
2003 & 5 & 3 & 6 & 4 & 8 & 17 & 28 & 23 & 19 & 21 & 26 & 10 & $\mathbf{1 7 1}$ \\
2004 & 3 & 5 & 5 & 8 & 10 & 11 & 12 & 29 & 24 & 24 & 24 & 8 & $\mathbf{1 6 3}$ \\
2005 & 6 & 4 & 8 & 3 & 7 & 13 & 9 & 27 & 29 & 55 & 39 & 26 & $\mathbf{2 2 9}$ \\
2006 & 5 & 2 & 1 & 3 & 12 & 4 & 16 & 16 & 37 & 39 & 23 & 12 & $\mathbf{1 7 0}$ \\
2007 & 8 & 3 & 2 & 1 & 5 & 14 & 14 & 20 & 59 & 35 & 28 & 33 & $\mathbf{2 2 2}$ \\
2008 & 7 & 2 & 1 & 6 & 6 & 7 & 13 & 24 & 26 & 60 & 25 & 35 & $\mathbf{2 1 2}$ \\
2009 & 13 & 5 & 6 & 6 & 9 & 14 & 11 & 44 & 20 & 57 & 33 & 38 & $\mathbf{2 5 6}$ \\
2010 & 12 & 5 & 4 & 5 & 7 & 21 & 14 & 39 & 77 & 42 & 18 & 26 & $\mathbf{2 7 0}$ \\
2011 & 11 & 1 & 3 & 3 & 6 & 12 & 14 & 35 & 49 & 29 & 35 & 22 & $\mathbf{2 2 1}$ \\
2012 & 7 & 5 & 1 & 3 & 6 & 10 & 10 & 44 & 46 & 60 & 40 & 14 & $\mathbf{2 4 6}$ \\
2013 & 12 & 4 & 4 & 3 & 7 & 2 & 7 & 30 & 24 & 41 & 18 & 23 & $\mathbf{1 7 4}$ \\
2014 & 5 & 3 & 2 & 1 & 5 & 9 & 19 & 14 & 41 & 31 & 27 & 30 & $\mathbf{1 8 8}$ \\
\hline mean & $\mathbf{7}$ & $\mathbf{3}$ & $\mathbf{4}$ & $\mathbf{4}$ & $\mathbf{7}$ & $\mathbf{1 1}$ & $\mathbf{1 5}$ & $\mathbf{2 8}$ & $\mathbf{3 6}$ & $\mathbf{3 8}$ & $\mathbf{2 6}$ & $\mathbf{2 3}$ & $\mathbf{2 0 0}$ \\
\hline
\end{tabular}

80 Table S4: Monthly and annual rainfall $\boldsymbol{P}_{\text {rain }}$ (in millimetres per month or millimetres per year) in the Qaidam Basin (QB) during the 14 hydrological years (2001-2014) covered by the HAR $10 \mathrm{~km}$ data set.

\begin{tabular}{rrrrrrrrrrrrrr}
\hline $\boldsymbol{P}_{\text {rain }}$ & 10 & 11 & 12 & 1 & 2 & 3 & 4 & 5 & 6 & 7 & 8 & 9 & year \\
\hline 2001 & 1 & 0 & 0 & 0 & 0 & 1 & 3 & 3 & 7 & 10 & 13 & 8 & $\mathbf{4 5}$ \\
2002 & 0 & 0 & 0 & 0 & 0 & 0 & 4 & 8 & 19 & 19 & 9 & 10 & $\mathbf{7 0}$ \\
2003 & 0 & 0 & 0 & 0 & 0 & 1 & 7 & 5 & 8 & 12 & 15 & 3 & $\mathbf{5 2}$ \\
2004 & 0 & 0 & 0 & 0 & 0 & 1 & 3 & 7 & 14 & 16 & 18 & 3 & $\mathbf{6 2}$ \\
2005 & 0 & 0 & 0 & 0 & 1 & 1 & 2 & 8 & 15 & 42 & 28 & 12 & $\mathbf{1 1 0}$ \\
2006 & 0 & 0 & 0 & 0 & 0 & 0 & 5 & 5 & 19 & 36 & 16 & 6 & $\mathbf{8 7}$ \\
2007 & 2 & 1 & 0 & 0 & 0 & 1 & 5 & 5 & 31 & 26 & 24 & 16 & $\mathbf{1 1 0}$ \\
2008 & 1 & 0 & 0 & 0 & 0 & 1 & 3 & 10 & 16 & 46 & 15 & 19 & $\mathbf{1 1 2}$ \\
2009 & 3 & 0 & 0 & 0 & 0 & 1 & 3 & 13 & 11 & 44 & 23 & 27 & $\mathbf{1 2 6}$ \\
2010 & 1 & 0 & 0 & 0 & 1 & 1 & 2 & 14 & 54 & 36 & 14 & 17 & $\mathbf{1 4 1}$ \\
2011 & 1 & 0 & 0 & 0 & 1 & 1 & 3 & 10 & 33 & 22 & 30 & 12 & $\mathbf{1 1 3}$ \\
2012 & 2 & 1 & 0 & 0 & 0 & 0 & 2 & 17 & 28 & 51 & 35 & 5 & $\mathbf{1 4 2}$ \\
2013 & 2 & 0 & 0 & 0 & 0 & 1 & 2 & 11 & 18 & 33 & 16 & 9 & $\mathbf{9 3}$ \\
2014 & 1 & 0 & 0 & 0 & 0 & 1 & 4 & 4 & 24 & 26 & 20 & 14 & $\mathbf{9 5}$ \\
\hline mean & $\mathbf{1}$ & $\mathbf{0}$ & $\mathbf{0}$ & $\mathbf{0}$ & $\mathbf{1}$ & $\mathbf{1}$ & $\mathbf{4}$ & $\mathbf{9}$ & $\mathbf{2 1}$ & $\mathbf{3 0}$ & $\mathbf{1 9}$ & $\mathbf{1 2}$ & $\mathbf{9 7}$ \\
\hline
\end{tabular}


Table S5: Monthly and annual snowfall $P_{\text {snow }}$ (in millimetres per month or millimetres per year) in the Qaidam Basin (QB) during the 14 hydrological years (2001-2014) covered by the HAR $10 \mathrm{~km}$ data set.

\begin{tabular}{rrrrrrrrrrrrrr}
\hline $\boldsymbol{P}_{\text {snow }}$ & 10 & 11 & 12 & 1 & 2 & 3 & 4 & 5 & 6 & 7 & 8 & 9 & year \\
\hline 2001 & 4 & 4 & 6 & 3 & 3 & 2 & 16 & 13 & 10 & 1 & 7 & 7 & $\mathbf{7 7}$ \\
2002 & 2 & 1 & 6 & 3 & 3 & 10 & 12 & 17 & 15 & 6 & 3 & 12 & $\mathbf{9 0}$ \\
2003 & 5 & 3 & 6 & 4 & 8 & 16 & 21 & 18 & 11 & 9 & 11 & 7 & $\mathbf{1 1 9}$ \\
2004 & 3 & 5 & 5 & 8 & 10 & 10 & 9 & 22 & 10 & 8 & 6 & 5 & $\mathbf{1 0 1}$ \\
2005 & 6 & 4 & 8 & 3 & 6 & 12 & 7 & 19 & 14 & 13 & 11 & 14 & $\mathbf{1 1 9}$ \\
2006 & 5 & 2 & 1 & 3 & 12 & 4 & 11 & 11 & 18 & 3 & 7 & 6 & $\mathbf{8 3}$ \\
2007 & 6 & 2 & 2 & 1 & 5 & 13 & 9 & 15 & 28 & 9 & 4 & 17 & $\mathbf{1 1 2}$ \\
2008 & 6 & 2 & 1 & 6 & 6 & 6 & 10 & 14 & 10 & 14 & 10 & 16 & $\mathbf{1 0 0}$ \\
2009 & 10 & 5 & 6 & 6 & 9 & 13 & 8 & 31 & 9 & 13 & 10 & 11 & $\mathbf{1 3 0}$ \\
2010 & 11 & 5 & 4 & 5 & 6 & 20 & 12 & 25 & 23 & 6 & 4 & 9 & $\mathbf{1 2 9}$ \\
2011 & 10 & 1 & 3 & 3 & 5 & 11 & 11 & 25 & 16 & 7 & 5 & 10 & $\mathbf{1 0 8}$ \\
2012 & 5 & 4 & 1 & 3 & 6 & 10 & 8 & 27 & 18 & 9 & 5 & 9 & $\mathbf{1 0 4}$ \\
2013 & 10 & 4 & 4 & 3 & 7 & 1 & 5 & 19 & 6 & 8 & 2 & 14 & $\mathbf{8 1}$ \\
2014 & 4 & 3 & 2 & 1 & 5 & 8 & 15 & 10 & 17 & 5 & 7 & 16 & $\mathbf{9 3}$ \\
\hline mean & $\mathbf{6}$ & $\mathbf{3}$ & $\mathbf{4}$ & $\mathbf{4}$ & $\mathbf{6}$ & $\mathbf{1 0}$ & $\mathbf{1 1}$ & $\mathbf{1 9}$ & $\mathbf{1 5}$ & $\mathbf{8}$ & $\mathbf{7}$ & $\mathbf{1 1}$ & $\mathbf{1 0 3}$ \\
\hline
\end{tabular}

Table S6: Monthly and annual actual evapotranspiration ET (in millimetres per month or millimetres per year) in the Qaidam Basin (QB) during the 14 hydrological years (2001-2014) covered by the HAR $10 \mathrm{~km}$ data set.

\begin{tabular}{crrrrrrrrrrrrr}
\hline $\boldsymbol{E} \boldsymbol{T}$ & 10 & 11 & 12 & 1 & 2 & 3 & 4 & 5 & 6 & 7 & 8 & 9 & year \\
\hline 2001 & 13 & 7 & 5 & 5 & 8 & 16 & 22 & 30 & 28 & 32 & 31 & 20 & $\mathbf{2 1 6}$ \\
2002 & 10 & 5 & 4 & 4 & 8 & 14 & 18 & 26 & 33 & 34 & 27 & 20 & $\mathbf{2 0 4}$ \\
2003 & 12 & 7 & 5 & 5 & 9 & 17 & 21 & 26 & 25 & 28 & 27 & 18 & $\mathbf{2 0 0}$ \\
2004 & 10 & 6 & 4 & 5 & 8 & 14 & 17 & 25 & 25 & 30 & 32 & 17 & $\mathbf{1 9 3}$ \\
2005 & 10 & 6 & 5 & 6 & 8 & 14 & 20 & 23 & 27 & 34 & 31 & 21 & $\mathbf{2 0 8}$ \\
2006 & 11 & 6 & 4 & 6 & 7 & 10 & 13 & 19 & 27 & 34 & 25 & 17 & $\mathbf{1 7 9}$ \\
2007 & 11 & 7 & 5 & 4 & 8 & 16 & 15 & 20 & 31 & 38 & 32 & 24 & $\mathbf{2 1 0}$ \\
2008 & 17 & 8 & 6 & 5 & 7 & 13 & 18 & 22 & 30 & 35 & 37 & 23 & $\mathbf{2 2 2}$ \\
2009 & 17 & 9 & 6 & 6 & 9 & 16 & 18 & 27 & 29 & 37 & 32 & 26 & $\mathbf{2 3 3}$ \\
2010 & 16 & 10 & 6 & 7 & 9 & 17 & 20 & 23 & 36 & 39 & 30 & 20 & $\mathbf{2 3 4}$ \\
2011 & 18 & 10 & 7 & 6 & 11 & 16 & 18 & 28 & 33 & 34 & 34 & 23 & $\mathbf{2 3 8}$ \\
2012 & 16 & 10 & 5 & 4 & 9 & 17 & 20 & 32 & 32 & 34 & 36 & 21 & $\mathbf{2 3 8}$ \\
2013 & 13 & 8 & 5 & 5 & 9 & 11 & 12 & 29 & 29 & 35 & 30 & 22 & $\mathbf{2 0 9}$ \\
2014 & 14 & 8 & 4 & 6 & 8 & 13 & 24 & 21 & 24 & 34 & 31 & 23 & $\mathbf{2 1 2}$ \\
\hline mean & $\mathbf{1 3}$ & $\mathbf{8}$ & $\mathbf{5}$ & $\mathbf{5}$ & $\mathbf{9}$ & $\mathbf{1 5}$ & $\mathbf{1 8}$ & $\mathbf{2 5}$ & $\mathbf{2 9}$ & $\mathbf{3 4}$ & $\mathbf{3 1}$ & $\mathbf{2 1}$ & $\mathbf{2 1 4}$ \\
\hline & & & & & & & & & & & &
\end{tabular}


90 Table S7: Monthly and annual water balance $\Delta S$ (in millimetres per month or millimetres per year) in the Qaidam Basin (QB) during the 14 hydrological years (2001-2014) covered by the HAR $10 \mathrm{~km}$ data set.

\begin{tabular}{|c|c|c|c|c|c|c|c|c|c|c|c|c|c|}
\hline$\Delta S$ & 10 & 11 & 12 & 1 & 2 & 3 & 4 & 5 & 6 & 7 & 8 & 9 & year \\
\hline 2001 & -8 & -3 & 1 & -2 & -5 & -13 & -3 & -13 & -11 & -21 & -11 & -5 & -94 \\
\hline 2002 & -8 & -4 & 2 & -1 & -4 & -4 & -2 & -2 & 2 & -9 & -15 & 1 & -44 \\
\hline 2003 & -6 & -4 & 1 & -1 & -1 & 0 & 7 & -2 & -6 & -7 & -1 & -8 & -29 \\
\hline 2004 & -7 & -1 & 1 & 3 & 2 & -3 & -5 & 3 & -1 & -6 & -8 & -9 & -30 \\
\hline 2005 & -4 & -2 & 3 & -3 & -2 & -1 & -11 & 4 & 2 & 21 & 8 & 5 & 21 \\
\hline 2006 & -6 & -4 & -2 & -2 & 5 & -6 & 3 & -3 & 10 & 5 & -3 & -5 & -10 \\
\hline 2007 & -3 & -4 & -3 & -4 & -3 & -2 & -2 & 0 & 29 & -2 & -4 & 10 & 11 \\
\hline 2008 & -10 & -7 & -4 & 1 & -1 & -7 & -5 & 2 & -5 & 25 & -12 & 12 & -10 \\
\hline 2009 & -5 & -4 & -1 & 0 & 0 & -1 & -7 & 17 & -9 & 20 & 1 & 12 & 24 \\
\hline 2010 & -4 & -5 & -3 & -2 & -2 & 4 & -6 & 16 & 42 & 3 & -13 & 6 & 36 \\
\hline 2011 & -7 & -9 & -3 & -3 & -5 & -4 & -4 & 6 & 16 & -5 & 1 & -1 & -17 \\
\hline 2012 & -9 & -5 & -4 & -1 & -3 & -7 & -10 & 12 & 14 & 26 & 3 & -7 & 8 \\
\hline 2013 & -1 & -4 & -2 & -2 & -2 & -9 & -5 & 1 & -5 & 6 & -12 & 1 & -35 \\
\hline 2014 & -9 & -6 & -3 & -4 & -3 & -4 & -5 & -6 & 17 & -3 & -4 & 7 & -24 \\
\hline mean & -6 & -4 & -1 & -2 & -2 & -4 & -4 & 2 & 7 & 4 & -5 & 1 & -14 \\
\hline
\end{tabular}

Table S8: Annual actual evapotranspiration ET in the Qaidam Basin (QB) during the calendar years 2001 to 2011 covered by both the HAR $10 \mathrm{~km}$ data set and the SEBS data as published in Jin et al. (2013).

\begin{tabular}{crrr}
\hline $\boldsymbol{E T} \mathbf{( m m} / \mathbf{a})$ & HAR $10 \mathrm{~km}$ & SEBS & Diff. \\
\hline 2001 & 211 & 73 & 138 \\
2002 & 208 & 74 & 134 \\
2003 & 196 & 78 & 118 \\
2004 & 194 & 85 & 110 \\
2005 & 205 & 144 & 61 \\
2006 & 181 & 123 & 58 \\
2007 & 219 & 135 & 84 \\
2008 & 222 & 145 & 77 \\
2009 & 232 & 170 & 62 \\
2010 & 236 & 169 & 67 \\
2011 & 234 & 182 & 52 \\
\hline $2001-2011$ & 213 & 125 & 87 \\
\hline $2001-2004$ & 202 & 77 & 125 \\
\hline $\mathbf{2 0 0 5 - 2 0 1 1}$ & $\mathbf{2 1 8}$ & $\mathbf{1 5 3}$ & $\mathbf{6 6}$ \\
\hline
\end{tabular}


Table S9: Mean monthly and annual air temperature $T$ (in deg C), specific humidity $q$ (in $\mathrm{g} / \mathrm{kg}$ ), precipitation $P$ (in millimetres per month or millimetres per year), rainfall $\boldsymbol{P}_{\text {rain }}$ (in millimetres per month or millimetres per year), snowfall $\boldsymbol{P}_{\text {snow }}$ (in millimetres per month or millimetres per year), actual evapotranspiration $E T$ (in millimetres per month or millimetres per year), and water balance

$100 \Delta \boldsymbol{S}=\boldsymbol{P}-\boldsymbol{E T}$ (in millimetres per month or millimetres per year) in the Qaidam Basin (QB) as in Table 1 but derived from the HAR $30 \mathrm{~km}$ data set; sigma: standard deviations of annual values for each quantity during the hydrological years 2001 to 2014.

\begin{tabular}{|c|c|c|c|c|c|c|c|c|c|c|c|c|c|c|}
\hline month & 10 & 11 & 12 & 1 & 2 & 3 & 4 & 5 & 6 & 7 & 8 & 9 & year & sigma \\
\hline$T$ & -0.9 & -8.6 & -13.2 & -14.8 & -10.6 & -5.9 & 0.1 & 5.2 & 9.8 & 12.3 & 11.5 & 6.7 & -0.7 & 0.6 \\
\hline$q$ & 2.3 & 1.4 & 1.1 & 1.0 & 1.3 & 1.5 & 2.0 & 2.9 & 4.4 & 5.8 & 5.3 & 4.0 & 2.8 & 0.2 \\
\hline$P$ & 8 & 4 & 4 & 4 & 7 & 11 & 15 & 29 & 39 & 39 & 28 & 23 & 210 & 45 \\
\hline$P_{\text {rain }}$ & 1 & 1 & 0 & 0 & 0 & 1 & 4 & 8 & 22 & 29 & 19 & 11 & 95 & 30 \\
\hline$P_{\text {snow }}$ & 7 & 3 & 4 & 4 & 7 & 10 & 11 & 21 & 17 & 10 & 9 & 12 & 115 & 20 \\
\hline$E T$ & 13 & 8 & 5 & 6 & 9 & 14 & 18 & 24 & 28 & 32 & 29 & 20 & 206 & 16 \\
\hline$\Delta S$ & -5 & -4 & -1 & -2 & -1 & -4 & -3 & 5 & 11 & 7 & -2 & 3 & 3 & 36 \\
\hline
\end{tabular}


\# Semi-empirical model for computation of water balance components in the

\# Qaidam Basin (QB) for different projections of lake extent (A.lake),

\# precipitation change in entire $\mathrm{QB}(\mathrm{dP} . \mathrm{QB})$ with respect to present-day

(HAR $10 \mathrm{~km}$ ), and mean rate of lake evaporation (ET.lake).

\# Regard: increased lake extent with high volumes of total lake evaporation

\# would lead to an additional increase in specific humidity, and thus enhance

\# local recycling of water. This feedback process is not considered in the

115 \# computations.

\# The $\mathrm{R}$ function $\mathrm{QB}$. $\mathrm{dS}$ provides estimates used in the 2 nd revison of the \# manuscript "Scherer, D.: Survival of the Qaidam Mega-Lake System under \# Mid-Pliocene Climates and its Restoration under Future Climates",

120 \# submitted to HESS in June 2020.

\# All quantitities are expressed as mean annual values. The following

\# abbreviations are used in the $\mathrm{R}$ code for naming of variables:

Quantities:

A: area (extent) $\left(\mathrm{km}^{\wedge} 2\right.$

P: precipitation ( $\mathrm{mm} / \mathrm{a})$

ET: actual evapotranspiration $(\mathrm{mm} / \mathrm{a})$

P. net: net precipitation (P - ET) ( $\mathrm{mm} / \mathrm{a})$

R: runoff from land areas into lakes (mm/a)

130

\# Subscripts:

Qaidam Basin (entire drainage basin)

PD: present-day (as represented in the HAR $10 \mathrm{~km}$ data set)

nml: no mega-lake projection (present-day lake extent as in HAR $10 \mathrm{~km}$ )

land: land area of $\mathrm{QB}$

low: low-altitude areas in the $\mathrm{QB}(\mathrm{z}<2.8 \mathrm{~km}$ a.s.l. $)$

140

Results of the computations are returned in a $R$ data frame.

\# Author: Dieter Scherer, Technische Universitaet Berlin, Germany

145

\# Iast update: 18.06 .2020

QB.dS <- function(A.lake, dP.QB, ET.lake) (

150

\# Quantities regarded not to change significantly over time

155

$\mathrm{A} . \mathrm{QB}<-254000 \# \mathrm{~km}^{\wedge} 2$; total area (A) of entire $Q B$

\# Sensitivities from HAR $10 \mathrm{~km}$ analysis

dET.dP.QB $<-0.2748$ \# sensitivity of ET to changes in $P$ averaged over entire $Q B$

ddS.dP.QB $<-0.7252$ \# sensitivity of $d S$ to changes in $P$ averaged over entire $Q B$

\# Quantities for present day (PD) from HAR $10 \mathrm{~km}$

165

A.lake.PD <- 1000 \# km^2; present-day lake area (no mega-lake system)

P.QB.PD <- 200 \# mm/a; P averaged over entire QB

P. low. PD <- $40 \#$ mm/a: P averaged over lower altitudes $(z<2.8 \mathrm{~km}$ a.s.l.)

ET.OB.PD <- 214 \# mm/a; ET averaged over entire $\mathrm{OB}$

dS.QB.PD <- P.QB.PD - ET.QB.PD \# mm/a; dS (= P.net) averaged over entire QB

175

\# Projected changes

A.land $<-\mathrm{A} . \mathrm{QB}-\mathrm{A} .1$ ake \# $\mathrm{km}^{\wedge} 2$; land area of $\mathrm{QB}$

\# Changes in $\mathrm{P}$ are assumed to uniformly take place at all altitudes

$\mathrm{P} . \mathrm{QB} \quad<-\mathrm{P} \cdot \mathrm{QB} \cdot \mathrm{PD}+\mathrm{dP} \cdot \mathrm{QB} \# \mathrm{~mm} / \mathrm{a} ; \mathrm{P}$ averaged over entire $\mathrm{QB}$

P. low <- P.low.PD + dP.QB \# mm/a; P averaged over lower altitudes $(<2.8 \mathrm{~km} \mathrm{a.s.l.}$

P.lake <- P.low \# mm/a; mega-lake system forms at lower altitudes

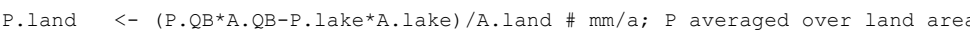


\# Changes for projection for QB with lake extent for PD (no mega-lake system)

$\mathrm{P} . \mathrm{QB} \cdot \mathrm{nml}<-\mathrm{P} . \mathrm{QB} \quad \# \mathrm{~mm} / \mathrm{a} ; \mathrm{P}$ averaged over entire $\mathrm{QB}$ $\mathrm{dS} \cdot \mathrm{QB} \cdot \mathrm{nml}<-\mathrm{dS} \cdot \mathrm{QB} \cdot \mathrm{PD}+\mathrm{ddS} \cdot \mathrm{dP} \cdot \mathrm{QB}^{*} \mathrm{dP} \cdot \mathrm{QB} \# \mathrm{~mm} / \mathrm{a}$; dS averaged over entire $\mathrm{QB}$ ET.QB.nml <- P.QB.nml - dS.QB.nml \# mm/a; ET averaged over entire $Q B$ $\# E T \cdot Q B \cdot n m l<-E T \cdot Q B \cdot P D+d E T \cdot d P \cdot Q^{*} d P \cdot Q B \# \mathrm{~mm} / a$; ET averaged over entire $Q B$

\# Changes in ET, net $P(P-E T)$ and $d S$

ET.land <- (ET.QB.nml*A.QB-ET.lake*A.lake.PD)/A.land \# mm/a; ET averaged over land area

$\mathrm{ET} . \mathrm{QB}<-\left(\mathrm{ET} . \mathrm{Cand}^{\star} \mathrm{A}\right.$.l land+ET.lake*A.lake)/A.QB \# mm/a; ET averaged over entire $\mathrm{QB}$

P.net.lake <- P.lake - ET.lake

P.net.land $<-$ P.land - ET.land

\# mm/a; P.net averaged over mega-lake system

P. net.QB $<-$ P.QB - ET.QB

\# $\mathrm{mm} / \mathrm{a}$; $;$ P. Pet averaged over land area

205

$\mathrm{dS} . \mathrm{QB} \quad<-\mathrm{P} \cdot$ net.QB $\quad \# \mathrm{~mm} / \mathrm{a} ; \mathrm{dS}$ (= P.net) averaged over entire $Q \mathrm{~B}$

\# Estimate change in (mega-) lake system

if (P.net.land $>=0)$

210 R.gW $<-0$ \# mm/a; no groundwater recharge (aquifers are considered to be filled) R.lake <- P.net.land*A.land/A.lake \# mm/a; runoff from land area into lakes

R.lake <- P.net.iand*A.land/A.iake

R.gw $\quad$ \# mm/a; all water losses are due to groundwater discharge

R.lake <- $\quad$ \# mm/a; no runoff from land area into lakes

dS.lake <- P.net.lake + R.lake \# mm/a; change in lake water storage

\# Return results as data frame

results <- data.frame (A.QB, A.land, A.lake, dP.QB, P.QB, P.land, P.lake,

ET.QB, ET.land, ET.lake, P.net.land, P.net.lake, dS.QB, R.lake, dS.lake)

225

return(results)

230

\# Values used in the Qaidam Basin study

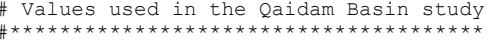

\# Projections for changes in precipitation

235

dP.QB.1 <- 100 \# mm/a; upper estimate from HAR analysis: projection 1

dP.QB.2 <- 50 \# mm/a; lower estimate from HAR analysis: projection 2

\# Projections for mean rates of lake evaporation

ET.lake.a <- 600 \# mm/a; lower estimate from HAR analysis (mean ET.lake in HAR: $650 \mathrm{~mm} / \mathrm{a}$ ): projection a

ET.lake.b <- 800 \# mm/a; medium estimate from literature and HAR analysis: projection b

240 ET.lake.c <- 1000 \# mm/a; upper estimate from HAR analysis: projection c

\# Approximate equilibrium requirements for changes in precipitation and mean rates of lake evaporation

dP.OB.mle.a <- 210 \# mm/a; required for sustaining maximum extent of mega-lake system (for ET.lake.a) $\mathrm{dP} . \mathrm{OB} \cdot \mathrm{mle} \cdot \mathrm{b}<-270 \mathrm{\#} \mathrm{mm/a}$; required for sustaining maximum extent of mega-lake system (for ET.lake.b) $\begin{array}{lll}\mathrm{dP} . \mathrm{QB} \cdot \mathrm{mle} . \mathrm{b} & <-270 \mathrm{\#} \mathrm{mm/a} \text {; required for sustaining maximum extent of mega-lake system (for ET.lake.b) } \\ \mathrm{dP} . \mathrm{QB} \cdot \mathrm{mle} . \mathrm{c} & <-330 \mathrm{\#} \mathrm{mm/a;} \text { required for sustaining maximum extent of mega-lake system (for ET.lake.C) }\end{array}$

dP.QB.HAR <- $19.3 \# \mathrm{~mm} / \mathrm{a}$; required for sustaining HAR lake extent (identical for ET.lake.a, ET.lake.b, and ET.lake.C)

250 ET.lake.mle.1 <- 260 \# mm/a; required for sustaining maximum extent of mega-lake system (for dP.QB.1) ET.lake.mle.2 <- 110 \# mm/a; required for sustaining maximum extent of mega-lake system (for dP.QB.2)

\# Lake extent either taken from HAR data and literature, or computed as equilibrium values by the semi-empirical model

A.lake.mle <- $59000 \# \mathrm{~km}^{\wedge} 2$; maximum extent of mega-lake system from literature

(HAR10: $z<=2786 \mathrm{~m}, \mathrm{~A}=59200 \mathrm{~km} \wedge 2$ ) A.lake.1a <- 25773 \# km^2; approximate sustainable mega-lake extent for projection $1 \mathrm{a}$ (HAR10: z<=2711 m, A = 25900 km^2) A.lake.1b <- 19580 \# km^2; approximate sustainable mega-lake extent for projection 1b (HAR10: z $<=2698 \mathrm{~m}, \mathrm{~A}=19700 \mathrm{~km} \wedge 2$ ) A.lake.1c <- $15864 \# \mathrm{~km}^{\wedge} 2$; approximate sustainable mega-lake extent for projection $1 \mathrm{C}$ (HAR10: $\mathrm{z}<=2688 \mathrm{~m}, \mathrm{~A}=15800 \mathrm{~km} \wedge 2$ ) A.lake.2a <- 10423 \# km^2; approximate sustainable mega-lake extent for projection 2a (HAR10: z<=2678 m, A = 10800 km^2)

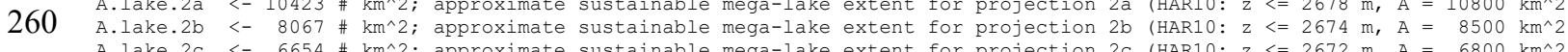
A.lake.2C <- $6654 \# \mathrm{~km}^{\wedge} 2$; approximate sustainable mega-lake extent for projection $2 \mathrm{C}$ (HAR10: $\mathrm{z}<=2672 \mathrm{~m}, \mathrm{~A}=6800 \mathrm{~km}$ (HAR10: A. lake.HAR <- $1000 \# \mathrm{~km}^{\wedge} 2$; present-day lake extent as in HAR $10 \mathrm{~km}$ (HAR10: $\left.z<=2654 \mathrm{~m}, A=1100 \mathrm{~km}^{\wedge} 2\right)$
$($ HAR10:

\# Example computation (as template for own computations)

results <- QB.dS (A.lake=A.lake.la, dP.QB=dP.QB.1, ET.lake=ET.lake.a) 